\title{
Progress in mesoscale science
}

\author{
J.L. Sarrao and G.W. Crabtree, Guest Editors \\ The domain of mesoscale science, where the discrete granularity of atoms and quantization of \\ energy give way to apparently continuous and infinitely divisible matter and energy, presents \\ a new frontier of scientific opportunity and yields new complex architectures, phenomena, \\ and functionalities. In this article, we describe some hallmarks of mesoscale science and \\ highlight research directions that are described in greater detail in subsequent articles in this \\ issue of MRS Bulletin. The exciting progress of the past several years and the rich unexplored \\ opportunities at the mesoscale offer extraordinary prospects for future advances.
}

\section{Introduction}

Over the last several decades and as frequently chronicled in the pages of MRS Bulletin, remarkable breakthroughs in nanoscale science have dramatically advanced our understanding of how materials work at the atomistic and quantum scales. These continuing fundamental advances at the nanoscale lay the foundation for understanding materials and phenomena at higher levels of complexity including, for example, defects, interfaces, heterogeneities, and composite structures, the domain of mesoscale science. ${ }^{1}$ A few years ago, we described the promise of mesoscale science in these pages. ${ }^{2}$ The remarkable progress made by the community in this short period of time demonstrates the rich untapped potential of the mesoscale., ${ }^{3,4}$

Mesoscale science, where atomic granularity, quantization of energy, and simplicity of structure and function give way to continuous matter and energy, complex structures, and composite functionalities, is a broad and rich horizon for innovative materials and chemistry research. ${ }^{1,2}$ The last half-century, and especially the last decade, has witnessed a remarkable drive to observations at ever finer length and time scales that reveal the atomic, molecular, and nanoscale origins of macroscopic behavior. This reductionist approach has led us to understand materials phenomena in terms of the smallest fundamental building blocks - atoms, ions, electrons, spins, and photons. As depicted graphically in Figure 1, the highly successful reductionist approach of the last 50 years lays the foundation for an equally rich journey in the opposite direction: A constructionist path that strategically combines the fundamental building blocks in new ways not found in nature, allowing new complexity and functionality to emerge at intermediate scales.

The conceptual shift from reductionism to constructionism in no way lessens the importance or significance of the nanoscale. Rather, it causes us to further challenge and deepen our understanding of materials and phenomena at all levels. The constructionist path, from atomic, molecular, and nanoscale, to greater complexity and higher functionality at larger scales, presents a qualitatively new feature: Entirely new mesoscale configurations of matter that lead to previously undiscovered macroscopic behavior, phenomena, and functionality. Reductionist science reveals a single pathway down from a given macroscopic behavior to its atomic, molecular, and nanoscale roots. In contrast, constructionist science embraces multiple and diverse pathways up from atomic, molecular, and nanoscale phenomena to meso- and macroscale behavior; many of which can lead to new macroscale outcomes that remain to be discovered and explored. This rich opportunity for discovering and controlling new macroscopic behavior by manipulating mesoscale architectures and emergent phenomena is the essence of mesoscale science.

The emergence of mesoscale behavior from nanoscale origins is marked by distinctive features in six attributes: the 


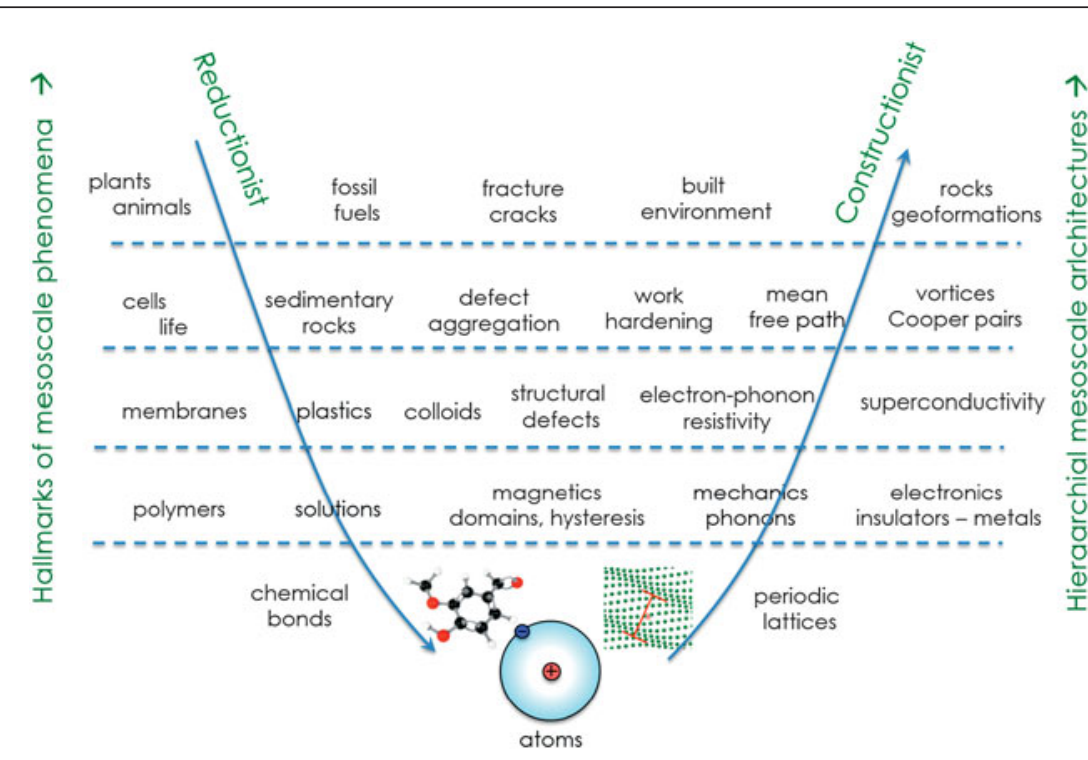

Figure 1. A schematic representation of the evolution from reductionist to constructionist approaches that is characteristic of mesoscale science.

mixtures of quasicrystalline and conventional order can be produced under certain conditions (Figure 2c), extending the variety and complexity of the superlattice behavior. Creating this kind of emergent behavior, understanding its origins in the competition of long-range and short-range interactions, and rationally controlling its mesoscale consequences are the first steps in intentionally designing new mesoscale functionality not found in nature.

The dynamic functionality of mesoscale materials is illustrated in Figure 3. ${ }^{6}$ The hydrogel poly $(N$-isopropylacrylamide) undergoes a hydrophobic to hydrophilic "swelling transition" between $32^{\circ} \mathrm{C}$ and $36^{\circ} \mathrm{C}$, taking on water and nearly doubling its volume. The degree of volume expansion depends on the nature and degree of cross-linking of the polymers making up the hydrogel. The volume transition can be converted to a variety of folding transitions by introducing gradients of cross-linking into the mesoscale structure at strategic locations.

diminishing importance of atomic granularity of matter; the transition from quantized to continuous energy; the collective behavior of many identical units; the interaction of disparate degrees of freedom; the appearance of defects, fluctuations, and statistical variation; and the heterogeneity in structure and dynamics. These hallmarks of mesoscale behavior affirm its presence and distinguish it from the adjacent realms of quantized nanoscale behavior at smaller scales and continuous macroscopic behavior at larger scales. ${ }^{1,2}$ Continued progress in our ability to control these mesoscale signatures requires advances in synthesis, characterization, theory and simulation, and especially, in their synergistic interaction.
Thick flat sections with full cross-linking form rigid panels connected by thin, partially cross-linked connectors that act as hinges. Rigid panels and flexible hinges can be patterned in a variety of shapes to form self-folding structures such as pyramids, rectangular boxes, or hand-like grippers that can capture and release cargo. Figure 3 shows the sequential selffolding with increasing temperature of pyramidal and rectangular boxes. By copolymerizing with other constituents, self-folding can be triggered by $\mathrm{pH}$, light, mechanical stress, or a magnetic field. The functionality of these smart, selffolding materials arises directly from the mesoscale design and integration of nanoscale polymer constituents, producing

\section{Mesoscale phenomena}

Many examples illustrate the richness and promise of mesoscale science. The emergence at the mesoscale of new structures and complexity not found in nature is illustrated in Figure 2. ${ }^{5}$ Mixtures of $9 \mathrm{~nm}$ Co nanoparticles and $4 \mathrm{~nm}$ $\mathrm{Ag}$ nanoparticles self-assemble into a diversity of superlattice structures, driven by the interplay of long-range magnetic and shortrange van der Waals interactions. At room temperature where the Co nanoparticles are ferromagnetic, long-range magnetic interactions mediate the collective response among Co nanoparticles, and the binary mixture selfassembles to a loosely packed quasicrystalline superlattice structure (Figure 2a), while at higher temperature where the Co nanoparticles are superparamagnetic, short-range van der Waals interactions create conventional densely packed periodic superlattice (Figure 2b). Two-phase

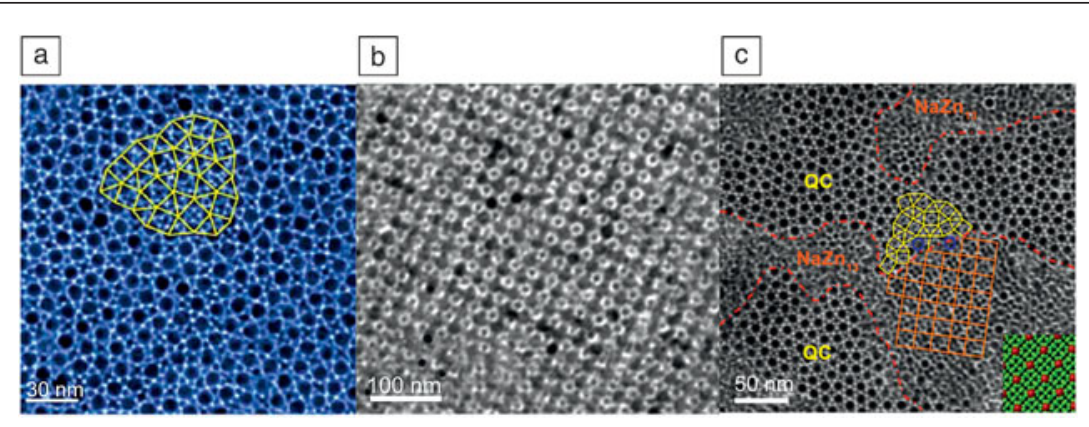

Figure 2. Transmission electron microscope images of superlattices of $9 \mathrm{~nm}$ Co and $4 \mathrm{~nm}$ Ag nanoparticles self-assembled from solvent evaporation. (a) Self-assembly at $25^{\circ} \mathrm{C}$, where the Co nanoparticles are ferromagnetic, exerting long-range magnetic interactions producing dodecagonal quasicrystalline order, illustrated by the square-triangular tiling shown in yellow. (b) Self-assembly at $65^{\circ} \mathrm{C}$, where the Co nanoparticles are superparamagnetic, long-range magnetic interactions are absent, and short-range van der Waals interactions dominate, producing a periodic $\mathrm{CoAg}_{3}$ structure. (c) Coexistence of quasicrystalline (QC) and periodic $\mathrm{CoAg}_{13}$ phases (the $\mathrm{CoAg}_{13}$ phase has the $\mathrm{NaZn}_{13}$ structure, as labeled) after self-assembly at $25^{\circ} \mathrm{C}$. Inset shows a model of the (100) plane of the $\mathrm{CoAg}_{13}$ structure, with Co nanoparticles in red and $\mathrm{Ag}$ nanoparticles in green. Reprinted with permission from Reference 5. (c) 2015 American Chemical Society. 


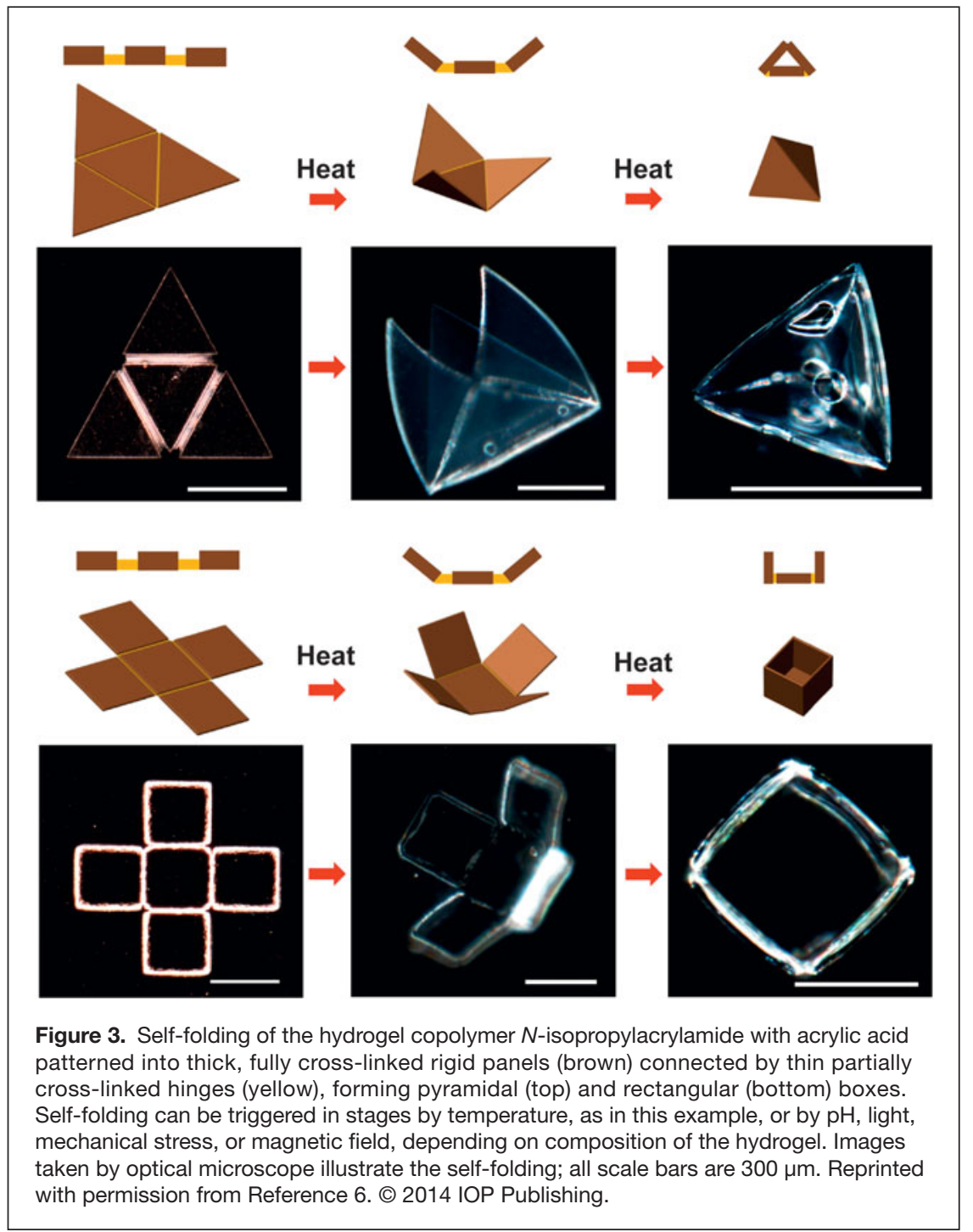

printing techniques. Finally, Rollett et al. discuss gaps in our ability to atomistically describe complex mesoscale systems, and thereby, highlight the importance of multimodal imaging closely coupled to modeling and simulation at the mesoscale, exploring the response of polycrystalline materials to thermal and mechanical loading as a representative challenge.

The emergence at the mesoscale of novel magnetic and superconducting ground states resulting from competing interactions has attracted attention for some time. As described by Basaran et al., the existence of multiple characteristic length scales for these interactions allows for the emergence of new phenomena when distinct characteristic scales become comparable to relevant geometric sizes. Topological interactions, geometric confinement, proximity between dissimilar materials, dimensional crossover, and collective effects induced by periodicity can all give rise to novel mesoscale phenomena. This article discusses how superconducting and magnetic materials serve as model systems, and insights from them can be extended to other coupled interactions in systems such as ferroelectrics and multiferroics where variations in characteristic length scales also enable tunability of competing interactions.

MacManus-Driscoll et al. demonstrate that self-assembled epitaxial oxide composite films represent a new hierarchical architecture in which high-quality mesoscale structures can be created. Previously, coupled electronic devices have been explored in such films, but only a narrow range of compositions and structure types were studied. ${ }^{7}$ Insufficient attention has

emergent phenomena not found in the bare constituents. The interaction of mechanical degrees of freedom leading to folding with chemical, thermal, optical, or magnetic degrees of freedom representing the trigger is a hallmark of mesoscale phenomena.

\section{In this issue}

Four articles demonstrate a subset of the excitement and diversity of mesoscale science. Basaran et al. highlight the role of competing interactions and how the balance among them is influenced by intrinsic and geometric/extrinsic length scales. They review several novel magnetic and superconducting ground states that emerge from mesoscale interactions. MacManus-Driscoll et al. and Raney and Lewis illustrate the key role of novel synthetic strategies in controlling mesoscale systems and hierarchical architectures. MacManus-Driscoll et al. describe recent advances in fabricating and manipulating self-assembled epitaxial oxide composite films, and Raney and Lewis highlight the importance of patterning materials in three dimensions at the mesoscale using a variety of $3 \mathrm{D}$ been paid to the wide range of possible materials combinations that could be included in these structures or to the novel materials functionalities that could result. They review the unique materials properties of these mesoscale composite structures, discussing their high crystallinity, structural uniformity, and orientation. In these systems, spatial ordering and characteristic layer separation can be used to tune structure and function. Metastable strain states can also be induced in these structures, and epitaxial stabilization can eliminate problems of surface degradation seen in bulk materials. Representative example systems such as room-temperature antiferromagneticferromagnetic coupling effects, interface-induced high-ionic conduction effects, and strongly enhanced ferroelectric properties demonstrate that composite films represent a novel approach to engineering new properties into mesoscale systems.

The ability to pattern materials in three dimensions can impact a wide array of structural, optical, electronic, and energy applications. As Raney and Lewis discuss in their article, three-dimensional printing allows one to design and rapidly 


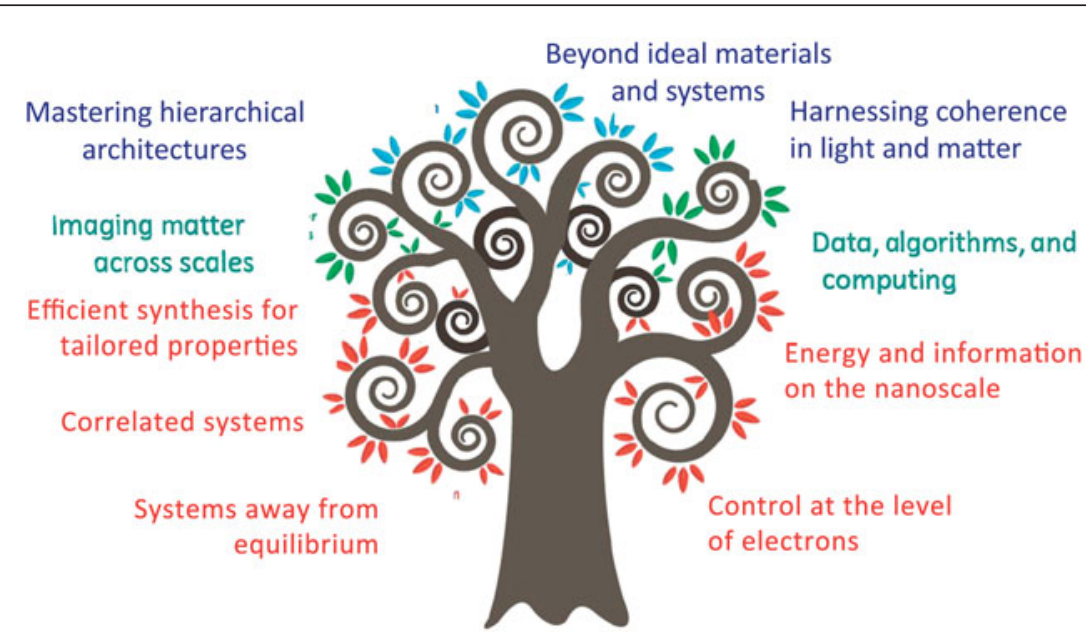

Figure 4. A graphical representation of the blossoming scientific opportunity spanned by the grand challenges (red) and transformative opportunities described in Reference 9.

\section{Future directions}

The evolution from reductionist atomistic approaches to constructionist mesoscale strategies is representative of a broader evolution and opportunity in materials research. In 2007, the US Department of Energy's Basic Energy Sciences Advisory Committee (BESAC) identified five grand challenges for science and the imagination. ${ }^{8}$ Despite significant progress and many exciting breakthroughs, these challenges remain unmet; however, they remain deeply stimulating. A number of these grand challenges ask reductionist questions about the fundamental interactions of atoms and molecules.

More recently, BESAC has revisited these challenges, asking what progress has been made in addressing them and whether new opportunities have emerged through the pursuit of the grand challenges. As a recent report

fabricate materials in complex shapes without the need for expensive tooling or lithographic strategies. The wide range of materials that can be printed and the ability to programmably control their architectures at the mesoscale represents a new design laboratory for novel materials functionality yielding unprecedented performance. These strategies hold the promise to impact not only structural assembly, but also multicomponent materials functionality.

Rollett et al. focus on imaging and modeling the response of polycrystalline materials to thermal and mechanical loading. In these systems, the relevant phenomena are too complex to be understood completely from first principles. However, both elastic and plastic responses of polycrystalline materials are becoming accessible through advanced high-energy x-ray characterization coupled with sophisticated data-intensive analysis. The combination of such diffraction experiments with computed tomography, for example, is producing new insights into how various microstructural features are related and coupled. As instrumentation improves in resolution and speed of readout, overall data rates and volumes are providing computational challenges, but with the benefit of producing enhanced spatial resolution. As an example, full-field, microstructure-resolved images provide ideal validation data for the corresponding micromechanical simulations with mesoscale fidelity. They discuss examples of this approach for tensile deformation of copper, grain growth in nickel and titanium, and fatigue cracks in superalloys, but these only scratch the surface of the potential impact of such coupled imaging and modeling approaches for understanding mesoscale phenomena.

Taken together, the articles in this issue validate the optimism that we expressed in $2012 .^{2}$ The mesoscale is proving to be a fertile landscape in which the evolution from quantized to continuous interactions give rise to novel phenomena and resulting functionality. concludes, ${ }^{9}$ the answer is a resounding yes. As depicted in Figure 4, the five grand challenges remain timely, engaging, and stimulating, and five additional transformative opportunities have emerged to further stimulate the community. An integrating theme across a number of these transformative opportunities is a constructionist vision of opportunities at the mesoscale and the means to seize those opportunities through advanced synthesis, imaging, and data analysis. Taken together, these directions are likely to further motivate the materials community for many years to come.

\section{Acknowledgments}

We thank our colleagues on the Mesoscale Science and Transformative Opportunities Subcommittees of the US Department of Energy Basic Energy Sciences Advisory Committee and acknowledge the authors of the articles that follow for their efforts in advancing the frontiers of mesoscale science and describing their work here. This work was supported in part by the Division of Materials Science and Engineering, Office of Basic Energy Sciences.

\section{References}

1. "From Quanta to the Continuum: Opportunities for Mesoscale Science," Report of US Department of Energy Basic Energy Sciences Advisory Committee, 2012.

2. G.W. Crabtree, J.L. Sarrao, MRS Bull. 37, 1079 (2012).

3. J.L. Sarrao, G.W. Crabtree, Curr. Opin. Solid State Mater. Sci. 19 (4), 201 (2015).

4. S. Yip, M.P. Short, Nat. Mater. 12, 774 (2013).

5. Z. Yang, J. Wei, P. Bonville, M.-P. Pilen, J. Am. Chem. Soc. 137, 4487 (2015). 6. C.K. Yoon, R. Xiao, J.H. Park, J. Cha, T.D. Nguyen, D.H. Gracias, Smart Mater. Struct. 23, 094008 (2014).

7. W. Zhang, R. Ramesh, J.L. MacManus-Driscoll, H. Wang, MRS Bull. 40, 736 (2015).

8. "Directing Matter and Energy: Five Challenges for Science and the Imagination," Report of US Department of Energy Basic Energy Sciences Advisory Committee, 2007

9. "Challenges at the Frontiers of Matter and Energy: Transformative Opportunities for Discovery Science," Report of US Department of Energy Basic Energy Sciences Advisory Committee, 2015. 